\title{
Pterulamides I-VI, New Linear Peptides from a Malaysian Pterula Species
}

Gerhard Lang, ${ }^{\dagger}$ Maya Mitova,${ }^{\dagger}$ Anthony L. J. Cole,${ }^{\ddagger}$ Laily Bin Din, ${ }^{\S}$ Sabaratnam

Vikineswary, ${ }^{\perp}$ Noorlidah Abdullah, ${ }^{\perp}$ John W. Blunt, ${ }^{\dagger}$ Murray H. G. Munro $*, \dagger$

\section{Supporting Information}

S1: $\quad{ }^{13} \mathrm{C},{ }^{1} \mathrm{H}, \mathrm{COSY}, \mathrm{HMBC}, \mathrm{TOCSY}$, and ROESY NMR data of pterulamide II (2; $500 \mathrm{MHz}$, in $\mathrm{CDCl}_{3}$ )

S2: ${ }^{13} \mathrm{C},{ }^{1} \mathrm{H}, \mathrm{COSY}, \mathrm{HMBC}, \mathrm{TOCSY}$, and ROESY NMR data of pterulamide III (3; $500 \mathrm{MHz}$, in $\mathrm{CDCl}_{3}$ )

S3: ${ }^{13} \mathrm{C},{ }^{1} \mathrm{H}, \mathrm{COSY}, \mathrm{HMBC}$, TOCSY, and ROESY NMR data of pterulamide IV (4; $500 \mathrm{MHz}$, in $\mathrm{CDCl}_{3}$ )

S4: ${ }^{13} \mathrm{C},{ }^{1} \mathrm{H}, \mathrm{COSY}, \mathrm{HMBC}, \mathrm{TOCSY}$, and ROESY NMR data of pterulamide VI $\left(6 ; 500 \mathrm{MHz}\right.$, in $\left.\mathrm{CDCl}_{3}\right)$

S5: $\quad{ }^{1} \mathrm{H}$ NMR spectrum of pterulamide I $\left(\mathbf{1} ; 500 \mathrm{MHz}\right.$ in $\left.\mathrm{MeOH}-d_{4}\right)$

S6: $\quad{ }^{1} \mathrm{H}$ NMR spectrum of pterulamide II $\left(2 ; 500 \mathrm{MHz}\right.$ in $\left.\mathrm{CDCl}_{3}\right)$

S7: $\quad{ }^{1} \mathrm{H}$ NMR spectrum of pterulamide III $\left(3 ; 500 \mathrm{MHz}\right.$ in $\left.\mathrm{CDCl}_{3}\right)$

S8: $\quad{ }^{1} \mathrm{H}$ NMR spectrum of pterulamide IV $\left(4 ; 500 \mathrm{MHz}\right.$ in $\left.\mathrm{CDCl}_{3}\right)$

S9: ${ }^{1} \mathrm{H}$ NMR spectrum of pterulamide $\mathrm{V}\left(\mathbf{5} ; 500 \mathrm{MHz}\right.$ in $\left.\mathrm{CDCl}_{3}\right)$

S10: ${ }^{1} \mathrm{H}$ NMR spectrum of pterulamide VI $\left(\mathbf{6} ; 500 \mathrm{MHz}\right.$ in $\left.\mathrm{CDCl}_{3}\right)$

\footnotetext{
* To whom correspondence should be addressed. Tel.: +64-3-3642434. Fax: +64-33642429. E-mail: murray.munro@ canterbury.ac.nz.

${ }^{\dagger}$ Department of Chemistry.

* School of Biological Sciences.

${ }^{\S}$ School of Chemical Sciences and Food Technology

${ }^{\perp}$ Institute of Biological Sciences
} 
Table S1: NMR data of pterulamide II (2; $500 \mathrm{MHz}$ in $\left.\mathrm{CDCl}_{3}\right)$ :

\begin{tabular}{|c|c|c|c|c|c|c|}
\hline Position & $\delta_{\mathrm{C}}^{\mathrm{a}}$ & $\delta_{\mathrm{H}}$ & COSY & HMBC & TOCSY & ROESY \\
\hline \multicolumn{7}{|l|}{$M T P^{b}$} \\
\hline $\mathrm{CO}$ & 165.9 & & & & & \\
\hline$\alpha$ & 111.1 & $6.05 d 14.6$ & $\beta$ & $\beta, \mathrm{CO}$ & $\beta$ & Ala-NMe, SMe \\
\hline$\beta$ & 147.9 & $7.80 d 14.6$ & $\alpha$ & $\alpha, \mathrm{CO}, \mathrm{SMe}$ & $\alpha$ & $\mathrm{SMe}$ \\
\hline $\mathrm{S}-\mathrm{Me}$ & 15.0 & $2.37 s$ & & $\beta$ & & $\alpha, \beta$ \\
\hline \multicolumn{7}{|l|}{ Ala } \\
\hline $\mathrm{CO}$ & 172.3 & & & & & \\
\hline$\alpha$ & 49.9 & $5.55 q 6.7$ & $\beta$ & $\mathrm{CO}$ & $\beta$ & $\mathrm{NMe}$, Ilel-NMe \\
\hline$\beta$ & 14.8 & $1.28 d 6.7$ & $\alpha$ & $\alpha, \mathrm{CO}$ & $\alpha$ & $\mathrm{NMe}$, Ile $1-\mathrm{NMe}$ \\
\hline$N-\mathrm{Me}$ & 30.9 & $3.04 s$ & & $\alpha, M T P-\mathrm{CO}$ & & $\alpha, \beta, M T P-\alpha$ \\
\hline \multicolumn{7}{|l|}{ Ile1 } \\
\hline $\mathrm{CO}$ & 171.7 & & & & & \\
\hline$\alpha$ & 57.4 & $5.21 d 10.7$ & $\beta$ & $\beta, \beta \mathrm{Me}, \mathrm{CO}$ & $\beta, \beta \mathrm{Me}, \gamma, \gamma^{\prime}, \delta$ & Vall-NMe, Vall- $\alpha, \mathrm{NMe}$ \\
\hline$\beta$ & 33.0 & $2.11 \mathrm{~m}$ & $\alpha, \gamma, \beta \mathrm{Me}$ & & $\alpha, \beta \mathrm{Me}, \gamma, \gamma^{\prime}, \delta$ & $\mathrm{NMe}$ \\
\hline$\beta-\mathrm{Me}$ & 15.7 & 0.86 & $\beta$ & $\alpha, \beta, \gamma$ & $\alpha, \beta, \gamma, \gamma^{\prime}, \delta$ & \\
\hline$\gamma$ & 24.3 & $1.28+0.99$ & $\beta, \delta$ & & $\alpha, \beta, \beta \mathrm{Me}, \delta$ & \\
\hline$\dot{\delta}$ & 10.6 & 0.85 & $\gamma$ & & $\alpha, \beta, \beta \mathrm{Me}, \gamma, \gamma^{\prime}$ & \\
\hline$N$-Me & 30.1 & $2.97 s$ & & $\alpha$, Ala-CO & & $\alpha, \beta$, Ala- $\alpha$, Ala- $\beta$ \\
\hline \multicolumn{7}{|l|}{ Vall } \\
\hline $\mathrm{CO}$ & 170.7 & & & & & \\
\hline$\alpha$ & 61.1 & $4.53 \mathrm{br}$ & $\beta$ & $\mathrm{CO}$ & $\beta, \gamma, \gamma^{\prime}$ & Ile $1-\alpha$ \\
\hline$\beta$ & 26.2 & $2.30 \mathrm{~m}$ & $\alpha, \gamma, \gamma^{\prime}$ & & $\alpha, \gamma, \gamma^{\prime}$ & $\mathrm{NMe}$ \\
\hline$\gamma$ & 19.2 & 0.86 & $\beta$ & $\beta, \gamma^{\prime}$ & $\alpha, \beta, \gamma^{\prime}$ & $\alpha, \mathrm{NMe}$ \\
\hline$\gamma^{\prime}$ & 18.4 & $0.74 d 6.3$ & $\beta$ & $\beta, \gamma$ & $\alpha, \beta, \gamma$ & $\mathrm{NMe}$ \\
\hline$N$-Me & 31.7 & $3.09 s$ & & Ile1-CO & & $\beta, \gamma, \gamma^{\prime}$, Ile $1-\alpha$ \\
\hline \multicolumn{7}{|l|}{ Val2 } \\
\hline $\mathrm{CO}$ & 173.4 & & & & & \\
\hline$\alpha$ & 54.3 & $4.63 t 8.3$ & $\beta, \mathrm{NH}$ & $\beta, \gamma, \gamma^{\prime}, \mathrm{CO}$ & $\beta, \gamma, \gamma^{\prime}$ & Ile 2-NMe \\
\hline$\beta$ & 30.9 & $2.01 \mathrm{~m}$ & $\alpha, \gamma+\gamma^{\prime}$ & & $\alpha, \gamma, \gamma^{\prime}$ & \\
\hline$\gamma+\gamma^{\prime}$ & 18.49 & 0.86 & $\beta$ & $\alpha, \beta$ & $\alpha, \beta$ & \\
\hline $\mathrm{NH}$ & & 7.25 & $\alpha$ & & $\alpha$ & \\
\hline \multicolumn{7}{|l|}{ Ile2 } \\
\hline $\mathrm{CO}$ & 170.5 & & & & & \\
\hline$\alpha$ & 61.4 & $4.68 d 11.5$ & $\beta$ & $\beta, \beta \mathrm{Me}, \mathrm{CO}$, Val2-CO & $\beta, \beta \mathrm{Me}, \gamma, \gamma^{\prime}, \delta$ & \\
\hline$\beta$ & 31.5 & $2.10 \mathrm{~m}$ & $\alpha, \gamma, \beta \mathrm{Me}$ & & $\alpha, \beta \mathrm{Me}, \gamma, \gamma^{\prime}, \delta$ & $\mathrm{NMe}, \mathrm{NHMe}$ \\
\hline$\beta-\mathrm{Me}$ & 18.5 & $0.89 m$ & $\beta$ & $\alpha, \beta, \gamma$ & $\alpha, \beta, \gamma, \gamma^{\prime}, \delta$ & \\
\hline$\gamma$ & 24.5 & $1.28+0.91$ & $\beta, \delta$ & & $\alpha, \beta, \beta \mathrm{Me}, \delta$ & \\
\hline$\dot{\delta}$ & 10.4 & 0.83 & $\gamma$ & & $\alpha, \beta, \beta \mathrm{Me}, \gamma, \gamma^{\prime}$ & \\
\hline$N-\mathrm{Me}$ & 31.1 & $3.16 s$ & & $\alpha$, Val2-CO & & $\beta$, NHMe, Val2- $\alpha$ \\
\hline $\mathrm{CONH}$ & & $6.82 b r$ & CONHMe & & CONHMe & \\
\hline CONHMe & 26.1 & $2.76 d 4.4$ & $\mathrm{CONH}$ & Ile2-CO & $\mathrm{CONH}$ & Ile2- $\beta$, Ile $2-\mathrm{NMe}$ \\
\hline
\end{tabular}

${ }^{13} \mathrm{C}$ shifts are from the HSQC-DEPT and HMBC spectra

${ }^{\mathrm{b}} \mathrm{MTP}=(E)$-3-methylthiopropenoyl residue 
Table S2: NMR data of pterulamide III (3; $500 \mathrm{MHz}$ in $\left.\mathrm{CDCl}_{3}\right)$ :

\begin{tabular}{|c|c|c|c|c|c|c|}
\hline Position & $\delta_{\mathrm{C}}{ }^{\mathrm{a}}$ & $\delta_{\mathrm{H}}$ & COSY & HMBC & TOCSY & ROESY \\
\hline \multicolumn{7}{|l|}{ Benzoyl } \\
\hline $\mathrm{CO}$ & 171.7 & & & & & \multirow{5}{*}{ Ala-NMe } \\
\hline 1 & 135.7 & & & & & \\
\hline 2,6 & 126.8 & $7.36 \mathrm{~m}$ & $3,4,5$ & 4 & $(3,5), 4$ & \\
\hline 3,5 & 128.6 & $7.41 \mathrm{~m}$ & $2,4,6$ & $1,(2,6)$ & $(2,6), 4$ & \\
\hline 4 & 129.8 & $7.41 \mathrm{~m}$ & $2,3,5,6$ & $(2,6),(3,5)$ & $(2,6),(3,5)$ & \\
\hline \multicolumn{7}{|l|}{ Ala } \\
\hline $\mathrm{CO}$ & 172.1 & & & & & \\
\hline$\alpha$ & 49.2 & $5.59 q 6.7$ & $\beta$ & & $\beta$ & $\mathrm{NMe}$, Ile1-NMe \\
\hline$\beta$ & 14.4 & $1.38 d 6.7$ & $\alpha$ & $\alpha, \mathrm{CO}$ & $\alpha$ & $\mathrm{NMe}$ \\
\hline$N-\mathrm{Me}$ & 33.1 & $2.96 s$ & & $\alpha, B z-\mathrm{CO}$ & & $\alpha, \beta, B z-2,6$ \\
\hline \multicolumn{7}{|l|}{ Ile1 } \\
\hline $\mathrm{CO}$ & 171.7 & & & & & \\
\hline$\alpha$ & 57.0 & $5.28 d 10.3$ & $\beta$ & $\beta, \mathrm{CO}$ & $\beta, \beta \mathrm{Me}, \gamma, \gamma^{\prime}, \delta$ & Vall-NMe, $\gamma$ \\
\hline$\beta$ & 32.9 & $2.19 \mathrm{~m}$ & $\alpha, \gamma, \beta-\mathrm{Me}$ & & $\alpha, \beta \mathrm{Me}, \gamma, \gamma^{\prime}, \delta$ & $\mathrm{NMe}$ \\
\hline$\beta-\mathrm{Me}$ & 15.7 & $0.87 \mathrm{~m}$ & $\beta$ & $\alpha, \beta, \gamma$ & $\alpha, \beta, \gamma, \gamma^{\prime}, \delta$ & \\
\hline$\gamma$ & 24.1 & $1.38,1.07 \mathrm{~m}$ & $\beta, \delta$ & & $\alpha, \beta, \beta \mathrm{Me}, \delta$ & $\alpha$ \\
\hline$\delta$ & 10.6 & $0.91 \mathrm{~m}$ & $\gamma$ & & $\alpha, \beta, \beta \mathrm{Me}, \gamma, \gamma$ & \\
\hline$N$-Me & 30.2 & $3.10 s$ & & $\alpha$ & & $\beta$, Ala- $\alpha$ \\
\hline \multicolumn{7}{|l|}{ Vall } \\
\hline $\mathrm{CO}$ & 170.5 & & & & & \\
\hline$\alpha$ & 61.1 & $4.57 \mathrm{br}$ & $\beta$ & $\mathrm{CO}$ & $\beta, \gamma, \gamma^{\prime}$ & $\gamma$ \\
\hline$\beta$ & 26.2 & $2.30 \mathrm{~m}$ & $\alpha, \gamma, \gamma^{\prime}$ & & $\alpha, \gamma, \gamma^{\prime}$ & $\mathrm{NMe}$ \\
\hline$\gamma$ & 19.3 & $0.86 m$ & $\beta$ & $\beta$ & $\alpha, \beta, \gamma^{\prime}$ & $\alpha, \mathrm{NMe}$ \\
\hline$\gamma^{\prime}$ & 18.2 & $0.76 d 6.3$ & $\beta$ & $\beta, \gamma$ & $\alpha, \beta, \gamma$ & $\mathrm{NMe}$ \\
\hline$N-\mathrm{Me}$ & 31.0 & $3.10 s$ & & Ile1-CO & & $\beta, \gamma, \gamma^{\prime}$, Ile $1-\alpha$ \\
\hline \multicolumn{7}{|l|}{ Val2 } \\
\hline $\mathrm{CO}$ & 173.0 & & & & & \\
\hline$\alpha$ & 53.8 & $4.67 \mathrm{~m}$ & $\beta, \mathrm{NH}$ & $\mathrm{CO}$ & $\beta, \gamma, \gamma^{\prime}$ & Ile 2-NMe \\
\hline$\beta$ & 31.1 & $1.99 \mathrm{~m}$ & $\alpha, \gamma, \gamma^{\prime}$ & & $\alpha, \gamma, \gamma^{\prime}$ & \\
\hline$\gamma$ & 24.5 & $0.86 m$ & $\beta$ & $\alpha, \beta, \gamma^{\prime}$ & $\alpha, \beta$ & \\
\hline$\gamma^{\prime}$ & 18.7 & $0.83 \mathrm{~m}$ & & $\beta, \gamma$ & & \\
\hline $\mathrm{NH}$ & & $7.05 \mathrm{br}$ & $\alpha$ & & & \\
\hline \multicolumn{7}{|l|}{ Ile2 } \\
\hline $\mathrm{CO}$ & 170.2 & & & & & \\
\hline$\alpha$ & 60.9 & $4.67 \mathrm{~m}$ & $\beta$ & $\mathrm{CO}$ & $\beta, \beta \mathrm{Me}, \gamma, \gamma^{\prime}, \delta$ & $\gamma$ \\
\hline$\beta$ & 31.1 & $2.10 \mathrm{~m}$ & $\alpha, \gamma, \beta-\mathrm{Me}$ & & $\alpha, \beta \mathrm{Me}, \gamma, \gamma^{\prime}, \delta$ & $\mathrm{NMe}$ \\
\hline$\beta \mathrm{Me}$ & 19.0 & $0.87 m$ & $\beta$ & $\alpha, \beta, \gamma$ & $\alpha, \beta, \gamma, \gamma, \delta$ & \\
\hline$\gamma$ & 24.2 & $1.30,0.99 \mathrm{~m}$ & $\beta, \delta$ & & $\alpha, \beta, \beta \mathrm{Me}, \delta$ & $\alpha$ \\
\hline$\delta$ & 10.4 & $0.81 \mathrm{~m}$ & $\gamma$ & & $\alpha, \beta, \beta \mathrm{Me}, \gamma, \gamma^{\prime}$ & \\
\hline $\mathrm{NMe}$ & 30.9 & $3.13 s$ & & $\alpha$, Val2-CO & & $\beta$, Val2- $\alpha$ \\
\hline $\mathrm{CONH}$ & & $6.49 \mathrm{br}$ & CONHMe & & CONHMe & \\
\hline CONHMe & 25.8 & $2.76 d 4.4$ & $\mathrm{CONH}$ & Ile2-CO & $\mathrm{CONH}$ & \\
\hline
\end{tabular}

${ }^{\mathrm{a}}{ }^{13} \mathrm{C}$ shifts are from the HSQC-DEPT and HMBC spectra 
Table S3: NMR data of pterulamide IV $\left(4 ; 500 \mathrm{MHz}\right.$ in $\left.\mathrm{CDCl}_{3}\right)$ :

\begin{tabular}{|c|c|c|c|c|c|c|}
\hline Position & $\delta_{\mathrm{C}}^{\mathrm{a}}$ & $\delta_{\mathrm{H}}$ & COSY & HMBC & TOCSY & ROESY \\
\hline \multicolumn{7}{|l|}{ Cinnamoyl } \\
\hline $\mathrm{CO}$ & 166.8 & & & & & \\
\hline$\alpha$ & 116.4 & $6.87 d 15.5$ & $\beta$ & $\mathrm{C}-1$ & $\beta$ & Ala-NMe, $(2,6)$ \\
\hline$\beta$ & 143.7 & $7.72 d 15.5$ & $\alpha$ & $\mathrm{C}-1$ & $\alpha$ & \\
\hline $\mathrm{C}-1$ & 137.7 & & & & & \\
\hline $\mathrm{C}-2,6$ & 127.7 & $7.53 \mathrm{~m}$ & $3,4,5$ & & $3,4,5$ & cinnamoyl- $\alpha$ \\
\hline C-3,5 & 128.7 & $7.37 \mathrm{~m}$ & $2,4,6$ & C-1 & $2,4,6$ & \\
\hline C-4 & 129.7 & $7.37 \mathrm{~m}$ & $2,3,5,6$ & & $2,3,5,6$ & \\
\hline \multicolumn{7}{|l|}{ Ala } \\
\hline $\mathrm{CO}$ & 172.1 & & & & & \\
\hline$\alpha$ & 49.1 & $5.67 q 7.1$ & $\beta$ & & $\beta$ & Ile1-NMe \\
\hline$\beta$ & 14.5 & $1.31 d 7.1$ & $\alpha$ & & $\alpha$ & $\mathrm{NMe}$ \\
\hline$N-\mathrm{Me}$ & 30.4 & $3.11 s$ & & $\alpha$, cinnamoyl-CO & & $\beta$, cinnamoyl $-\alpha$ \\
\hline \multicolumn{7}{|l|}{ Ile $1^{b}$} \\
\hline $\mathrm{CO}$ & 171.7 & & & & & \\
\hline$\alpha$ & 56.9 & $5.25 d 11.1$ & $\beta$ & & $\beta, \beta \mathrm{Me}, \gamma, \gamma^{\prime}, \delta$ & Vall-NMe \\
\hline$\beta$ & 32.6 & $2.16 \mathrm{~m}$ & $\alpha, \gamma, \beta-\mathrm{Me}$ & & $\alpha, \beta \mathrm{Me}, \gamma, \gamma^{\prime}, \delta$ & $\mathrm{NMe}$ \\
\hline$\beta-\mathrm{Me}$ & 15.4 & $0.86 m$ & $\beta$ & $\alpha, \beta, \gamma$ & $\alpha, \beta, \gamma, \gamma^{\prime}, \delta$ & \\
\hline$\gamma$ & 23.9 & $1.31 m+1.02$ & $\beta, \delta$ & & $\alpha, \beta, \beta \mathrm{Me}, \delta$ & $\mathrm{NMe}$ \\
\hline$\delta$ & 10.3 & 0.86 & $\gamma$ & & $\alpha, \beta, \beta \mathrm{Me}, \gamma, \gamma^{\prime}$ & \\
\hline$N$-Me & 29.9 & $3.00 s$ & & $\alpha$, Ala-CO & & $\beta, \gamma$, Ala $-\alpha$ \\
\hline \multicolumn{7}{|l|}{ Vall $^{b}$} \\
\hline \multicolumn{7}{|l|}{$\mathrm{CO}$} \\
\hline$\alpha$ & 60.6 & $4.43 \mathrm{br}$ & $\beta$ & & $\beta, \gamma, \gamma^{\prime}$ & \\
\hline$\beta$ & 25.9 & $2.33 \mathrm{~m}$ & $\alpha, \gamma, \gamma^{\prime}$ & & $\alpha, \gamma, \gamma^{\prime}$ & $\mathrm{NMe}$ \\
\hline$\gamma$ & 19.3 & $0.87 m$ & $\beta$ & $\beta, \gamma^{\prime}$ & $\alpha, \beta, \gamma^{\prime}$ & \\
\hline$\gamma^{\prime}$ & 17.9 & $0.76 d 6.7$ & $\beta$ & $\beta, \gamma$ & $\alpha, \beta, \gamma$ & $\mathrm{NMe}$ \\
\hline$N-\mathrm{Me}$ & 30.5 & $3.08 s$ & & Ile1-CO & & $\beta, \gamma^{\prime}$, Ilel- $\alpha$ \\
\hline \multicolumn{7}{|l|}{$\operatorname{Val} 2^{b}$} \\
\hline $\mathrm{CO}$ & 173.0 & & & & & \\
\hline$\alpha$ & 53.4 & $4.67 t 8.5$ & $\beta, \mathrm{NH}$ & & $\beta, \gamma, \gamma^{\prime}$ & Ile 2-NMe \\
\hline$\beta$ & 30.7 & $1.98 \mathrm{~m}$ & $\alpha, \gamma, \gamma^{\prime}$ & & $\alpha, \gamma, \gamma^{\prime}$ & \\
\hline$\gamma$ & 17.6 & 0.84 & $\beta$ & $\alpha, \beta$ & $\alpha, \beta$ & \\
\hline$\gamma^{\prime}$ & 15.4 & 0.84 & $\beta$ & $\alpha, \beta$ & $\alpha, \beta$ & \\
\hline $\mathrm{NH}$ & & $6.70 \mathrm{br}$ & $\alpha$ & & & \\
\hline \multicolumn{7}{|l|}{$I l e 2^{b}$} \\
\hline $\mathrm{CO}$ & 171.0 & & & & & \\
\hline$\alpha$ & 60.7 & $4.60 d 11.1$ & $\beta$ & & $\beta, \beta \mathrm{Me}, \gamma, \gamma^{\prime}, \delta$ & \\
\hline$\beta$ & 31.1 & $2.12 \mathrm{~m}$ & $\alpha, \gamma, \beta \mathrm{Me}$ & & $\alpha, \beta \mathrm{Me}, \gamma, \gamma^{\prime}, \delta$ & $\mathrm{NMe}$ \\
\hline$\beta-\mathrm{Me}$ & 18.8 & $0.87 \mathrm{~m}$ & $\beta$ & $\alpha, \beta, \gamma$ & $\alpha, \beta, \gamma, \gamma^{\prime}, \delta$ & \\
\hline$\gamma$ & 23.8 & $1.28 m+0.94$ & $\beta, \delta$ & & $\alpha, \beta, \beta \mathrm{Me}, \delta$ & \\
\hline$\delta$ & 10.1 & 0.81 & $\gamma$ & & $\alpha, \beta, \beta \mathrm{Me}, \gamma, \gamma^{\prime}$ & \\
\hline$N$-Me & 30.4 & $3.10 \mathrm{~s}$ & & $\alpha$, Val2-CO & & $\beta$, Val2- $\alpha$ \\
\hline $\mathrm{CON} H^{\mathrm{d}}$ & & $6.22 \mathrm{br}$ & CONHMe & & $\mathrm{NH} M e$ & \\
\hline CONHMe & 25.5 & $2.76 d 4.8$ & $\mathrm{CONH}$ & Ile2-CO & $\mathrm{NH}$ & \\
\hline
\end{tabular}

\footnotetext{
${ }^{\mathrm{a} 13} \mathrm{C}$ shifts from HSQC-DEPT and HMBC spectra
}

${ }^{\mathrm{b}}$ similar amino acids are numbered consecutively from $N$ - to $C$-terminal end 
Table S4: NMR data of pterulamide VI $\left(6 ; 500 \mathrm{MHz}\right.$ in $\left.\mathrm{CDCl}_{3}\right)$ :

\begin{tabular}{|c|c|c|c|c|c|c|}
\hline Position & $\delta_{\mathrm{C}}{ }^{\mathrm{a}}$ & $\delta_{\mathrm{H}}$ & COSY & HMBC & ROESY & TOCSY \\
\hline \multicolumn{7}{|l|}{$B z$} \\
\hline $\mathrm{CO}$ & 172.0 & & & & & \\
\hline 1 & 136.4 & & & & & \\
\hline $2 / 6$ & 126.6 & $7.29 \mathrm{~m}$ & $3 / 5$ o. 4 & $\mathrm{CO}, 2 / 6,4$ & Vall-NMe & \\
\hline $3 / 5$ & 128.8 & 7.40 & $2 / 6$ & $2 / 6,3 / 5$ & & \\
\hline 4 & 129.7 & 7.40 & $2 / 6$ & $2 / 6,3 / 5$ & & \\
\hline \multicolumn{7}{|l|}{ Vall $^{b}$} \\
\hline $\mathrm{CO}$ & 170.6 & & & & & \\
\hline$\alpha$ & 58.4 & $5.33 d 11.0$ & $\beta$ & $\mathrm{CO}, \beta, \gamma, \gamma^{\prime}, \mathrm{NMe}, B z-\mathrm{CO}$ & $\beta, \gamma, \gamma^{\prime}$ & $\beta, \gamma, \gamma^{\prime}$ \\
\hline$\beta$ & 26.9 & 2.43 & $\alpha, \gamma, \gamma^{\prime}$ & $\alpha$ & $\alpha, \gamma, \gamma^{\prime}$ & $\alpha, \gamma, \gamma^{\prime}$ \\
\hline$\gamma$ & 19.6 & 0.93 & $\beta$ & $\alpha, \beta, \gamma^{\prime}$ & $\alpha, \beta$ & $\alpha, \beta$ \\
\hline$\gamma^{\prime}$ & 18.2 & $0.98 d 6.5$ & $\beta$ & $\alpha, \beta, \gamma$ & $\alpha, \beta$ & $\alpha, \beta$ \\
\hline$N-\mathrm{Me}$ & 32.9 & $2.87 s$ & & $\alpha, B z-\mathrm{CO}$ & $B z-2 / 6$ & \\
\hline \multicolumn{7}{|l|}{$\operatorname{Val}^{b}$} \\
\hline $\mathrm{CO}$ & 171.0 & & & & & \\
\hline$\alpha$ & 58.6 & $5.22 d 11.0$ & $\beta$ & $\mathrm{CO}, \beta, \gamma, \gamma^{\prime}, \mathrm{NMe}$ & $\begin{array}{l}\text { Val3-NMe, } \\
\beta, \gamma, \gamma^{\prime}\end{array}$ & $\beta, \gamma, \gamma^{\prime}$ \\
\hline$\beta$ & 27.3 & 2.42 & $\alpha, \gamma, \gamma^{\prime}$ & & $\beta, \gamma, \gamma^{\prime}$ & $\beta, \gamma, \gamma^{\prime}$ \\
\hline$\gamma$ & 19.9 & 0.90 & $\beta$ & $\alpha, \beta, \gamma^{\prime}$ & $\alpha, \gamma, \gamma^{\prime}$ & $\alpha, \gamma, \gamma^{\prime}$ \\
\hline$\gamma$ & 17.9 & 0.84 & $\beta$ & $\alpha, \beta, \gamma$ & $\alpha, \beta$ & $\alpha, \beta$ \\
\hline$N$-Me & 30.6 & $3.11 s$ & & $\alpha$, Vall-CO & & \\
\hline \multicolumn{7}{|l|}{$\operatorname{Val} 3^{b}$} \\
\hline $\mathrm{CO}$ & 169.5 & & & & & \\
\hline$\alpha$ & 62.7 & 4.58 & $\beta$ & $\mathrm{CO}, \beta, \gamma, \gamma^{\prime}, \mathrm{NMe}$ & $\begin{array}{l}\text { Val4-NH, } \\
\beta, \gamma, \gamma^{\prime}\end{array}$ & $\beta, \gamma, \gamma^{\prime}$ \\
\hline$\beta$ & 26.0 & 2.26 & $\alpha, \gamma, \gamma^{\prime}$ & & $\alpha, \gamma, \gamma^{\prime}$ & $\alpha, \gamma, \gamma^{\prime}$ \\
\hline$\gamma$ & 18.4 & $0.74 d 6.5$ & $\beta$ & $\alpha, \beta, \gamma^{\prime}$ & $\alpha, \beta$ & $\alpha, \beta$ \\
\hline$\gamma$ & 19.5 & 0.89 & $\beta$ & $\alpha, \beta, \gamma$ & $\alpha, \beta$ & $\alpha, \beta$ \\
\hline$N-\mathrm{Me}$ & 30.6 & $3.04 s$ & & $\alpha$, Val2-CO & Val2- $\alpha$ & \\
\hline \multicolumn{7}{|l|}{ Val4 ${ }^{b}$} \\
\hline $\mathrm{CO}$ & 172.4 & & & & & \\
\hline$\alpha$ & 53.8 & $4.71 t 9.0$ & $\beta, \mathrm{NH}$ & $\mathrm{CO}, \beta, \gamma$ & $\beta, \gamma, \gamma^{\prime}$ & $\mathrm{NH}, \beta, \gamma, \gamma^{\prime}$ \\
\hline$\beta$ & 31.1 & $1.95 \mathrm{~m}$ & $\alpha, \gamma, \gamma^{\prime}$ & & $\alpha, \gamma, \gamma^{\prime}$ & $\mathrm{NH}, \alpha, \gamma, \gamma^{\prime}$ \\
\hline$\gamma$ & 17.8 & 0.82 & $\beta$ & $\alpha, \beta$ & $\alpha, \beta$ & $\mathrm{NH}, \alpha, \beta$ \\
\hline$\gamma^{\prime}$ & $\sim 18$ & 0.85 & $\beta$ & $\alpha, \beta, \gamma$ & $\alpha, \beta$ & $\mathrm{NH}, \alpha, \beta$ \\
\hline $\mathrm{NH}$ & & $6.54 d 9.0$ & $\alpha$ & Val3-CO & Val3- $\alpha$ & $\alpha, \beta, \gamma, \gamma^{\prime}$ \\
\hline \multicolumn{7}{|l|}{ Ile } \\
\hline $\mathrm{CO}$ & 171.8 & & & & & \\
\hline$\alpha$ & 56.5 & $5.28 d 11.0$ & $\beta$ & $\mathrm{CO}, \beta, \beta-\mathrm{Me}, \mathrm{NMe}$ & $\beta, \beta-\mathrm{Me}$ & $\beta, \beta-\mathrm{Me}, \gamma$ \\
\hline$\beta$ & 33.5 & $2.14 m$ & $\alpha, \beta-\mathrm{Me}$ & & $\alpha, \gamma$ & $\alpha, \beta-\mathrm{Me}, \gamma$ \\
\hline$\beta-\mathrm{Me}$ & 15.3 & 0.87 & $\beta$ & $\alpha, \beta, \gamma$ & $\alpha$ & $\alpha, \beta$ \\
\hline$\gamma$ & 24.1 & $0.94+1.25 \mathrm{~m}$ & & & $\beta$ & \\
\hline$\dot{\delta}$ & 10.7 & 0.82 & & $\beta, \gamma$ & & \\
\hline$N$-Me & 30.7 & $3.10 s$ & & $\alpha$, Val4-CO & & \\
\hline \multicolumn{7}{|l|}{ Val5 $^{b}$} \\
\hline $\mathrm{CO}$ & 171.4 & & & & & \\
\hline$\alpha$ & 63.9 & 4.65 & $\beta$ & $\mathrm{CO}$ & $\beta, \gamma, \gamma^{\prime}$ & $\beta, \gamma, \gamma^{\prime}$ \\
\hline$\beta$ & 26.9 & 2.30 & $\alpha, \gamma, \gamma^{\prime}$ & & $\alpha, \gamma, \gamma^{\prime}$ & $\alpha, \gamma, \gamma^{\prime}$ \\
\hline$\gamma$ & 18.9 & 0.79 & $\beta$ & $\alpha, \beta, \gamma^{\prime}$ & $\alpha, \beta$ & $\alpha, \beta$ \\
\hline$\gamma^{\prime}$ & 19.5 & $1.05 d 6.0$ & $\beta$ & $\alpha, \beta, \gamma$ & $\alpha, \beta$ & $\alpha, \beta$ \\
\hline$N-\mathrm{Me}$ & 33.1 & $3.10 s$ & & $\alpha$, Ile-CO & & \\
\hline
\end{tabular}

${ }^{\mathrm{a} 13} \mathrm{C}$ shifts from HSQC-DEPT and HMBC spectra

${ }^{\mathrm{b}}$ similar amino acids are numbered consecutively from $N$ - to $C$-terminal end 
S5: ${ }^{1} \mathrm{H}$ NMR of pterulamide I $\left(\mathbf{1} ; 500 \mathrm{MHz}\right.$, in $\left.\mathrm{MeOH}-d_{4}\right)$




S6: ${ }^{1} \mathrm{H}$ NMR of pterulamide II (2; $\left.500 \mathrm{MHz}, \mathrm{inCDCl}_{3}\right)$

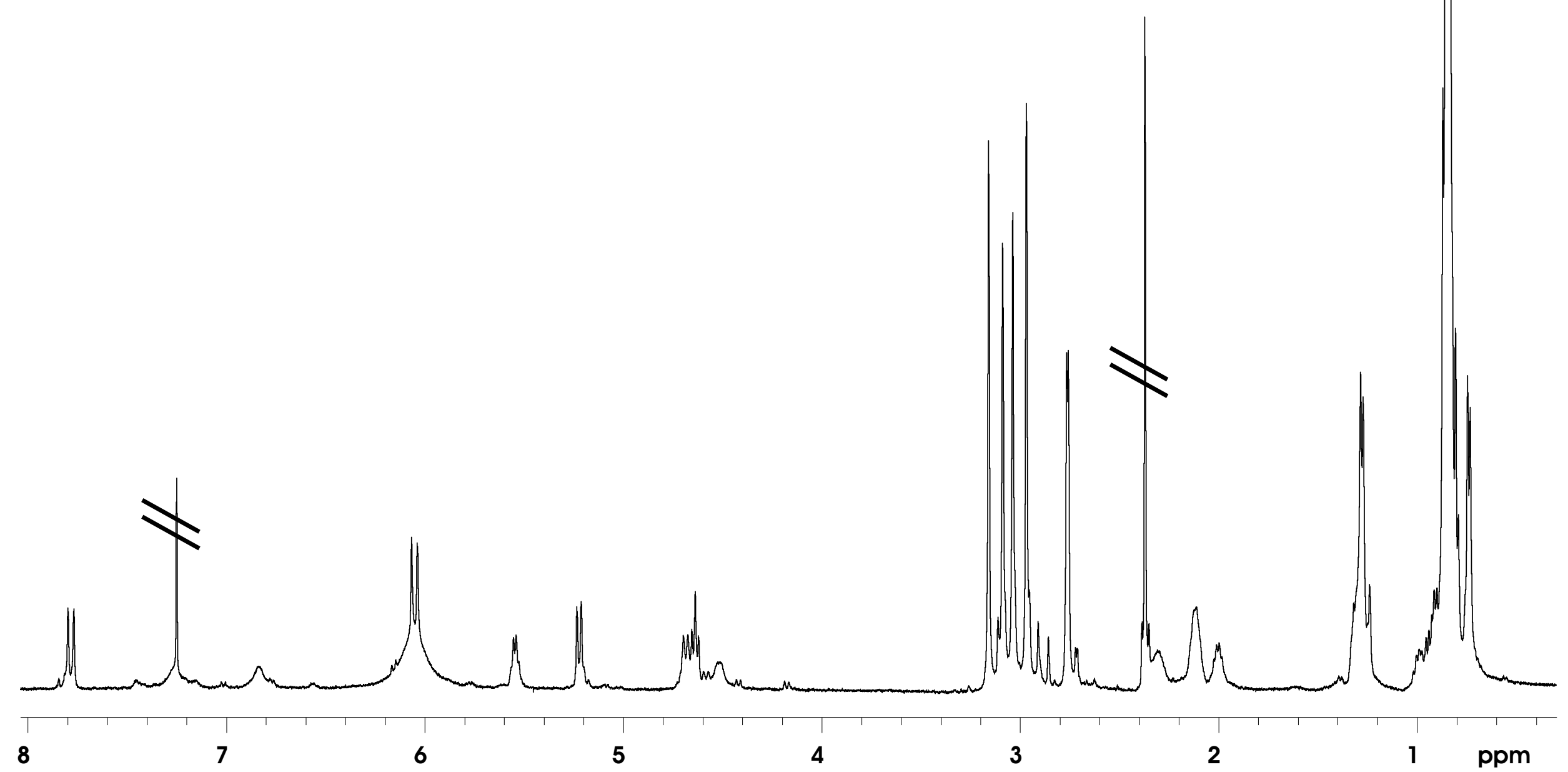


S7: ${ }^{1} \mathrm{H}$ NMR of pterulamide III $\left(3 ; 500 \mathrm{MHz}\right.$, inCDCl $\left._{3}\right)$

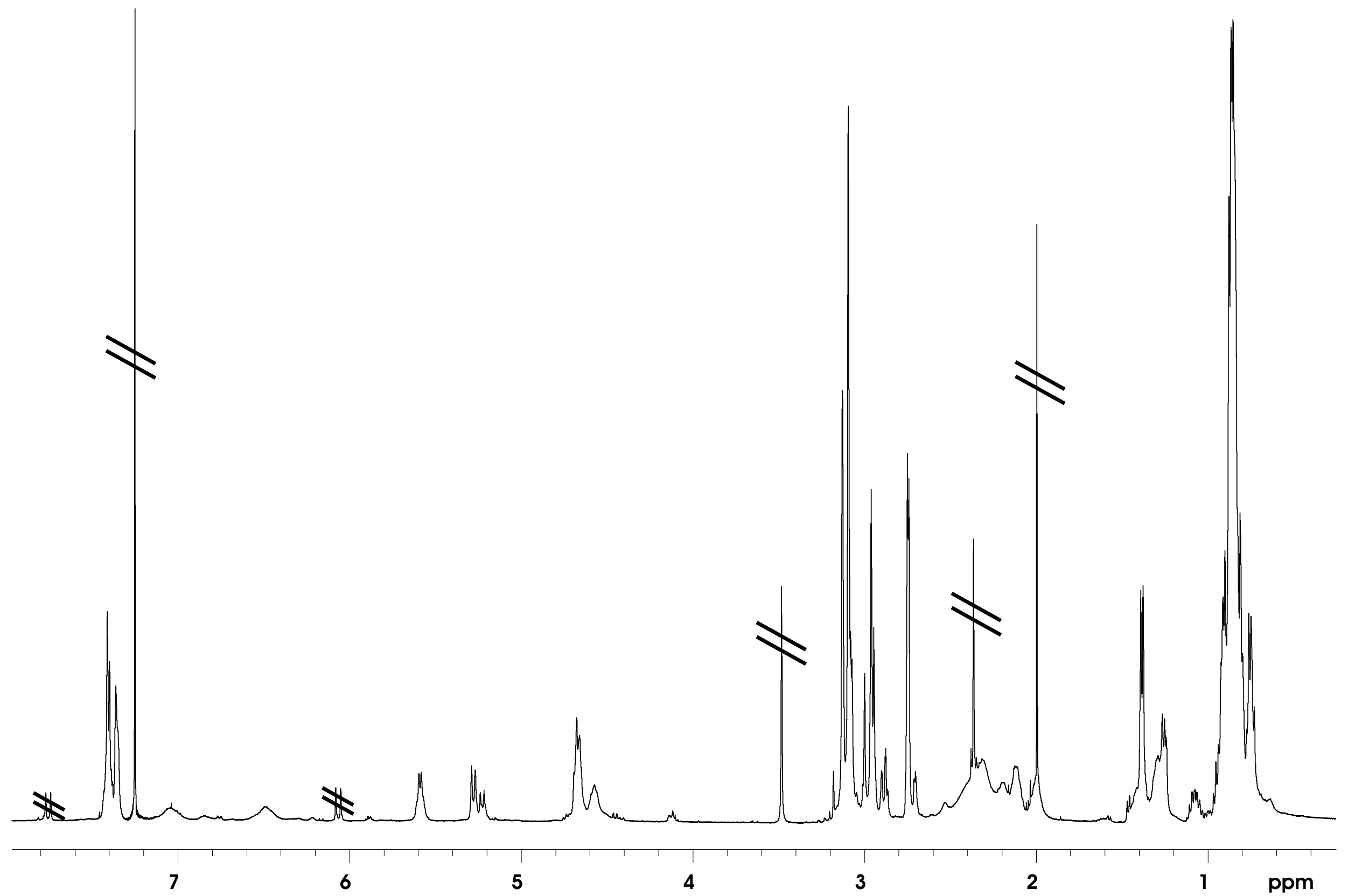


S8: ${ }^{1} \mathrm{H}$ NMR of pterulamide IV $\left(4 ; 500 \mathrm{MHz}, \mathrm{inCDCl}_{3}\right)$

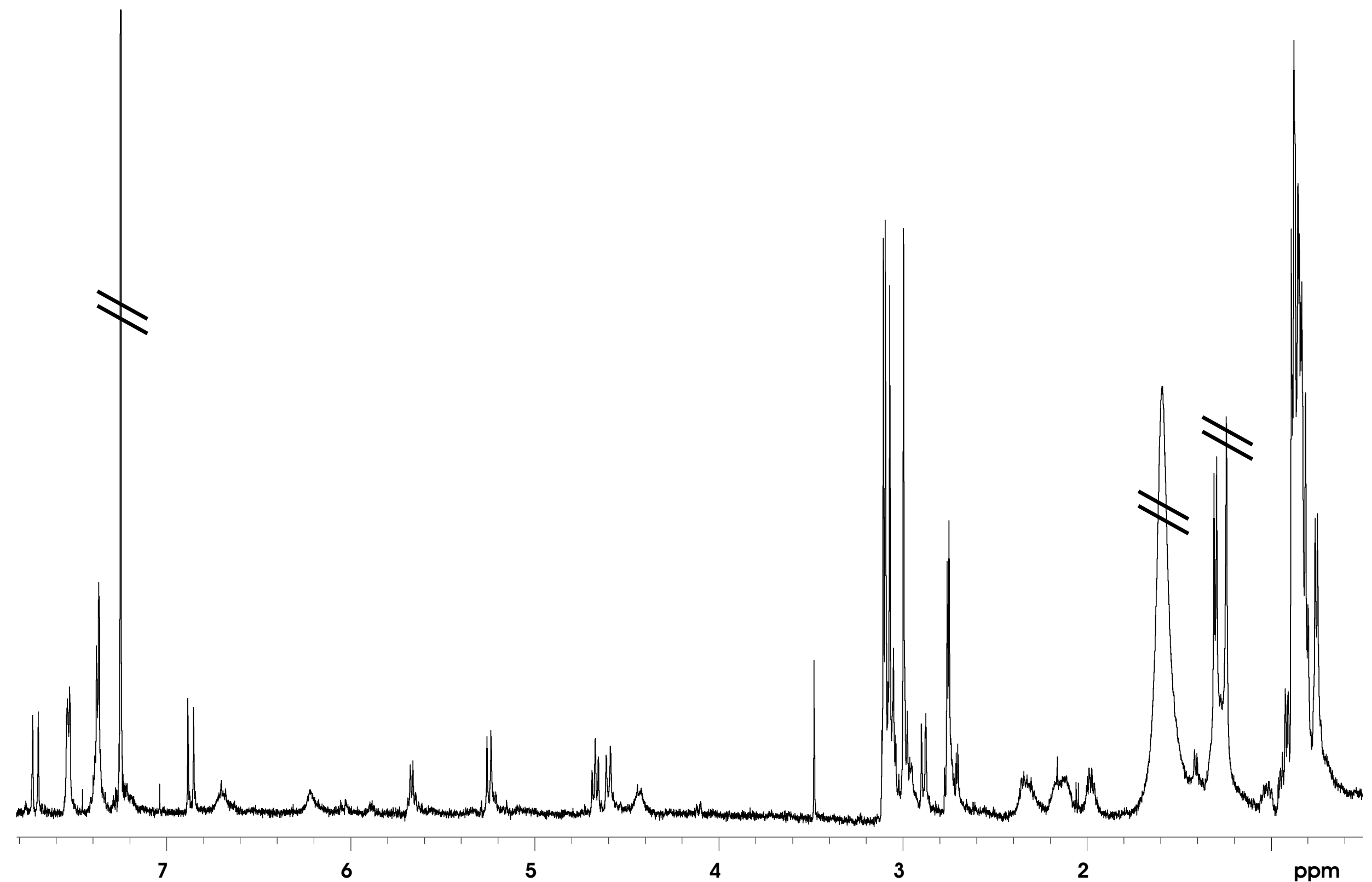


S9: ${ }^{1} \mathrm{H}$ NMR of pterulamide V $\left(\mathbf{5} ; 500 \mathrm{MHz}, \mathrm{inCDCl}_{3}\right)$




S10: ${ }^{1} \mathrm{H}$ NMR of pterulamide VI $\left(\mathbf{6} ; 500 \mathrm{MHz}, \mathrm{inCDCl}_{3}\right)$

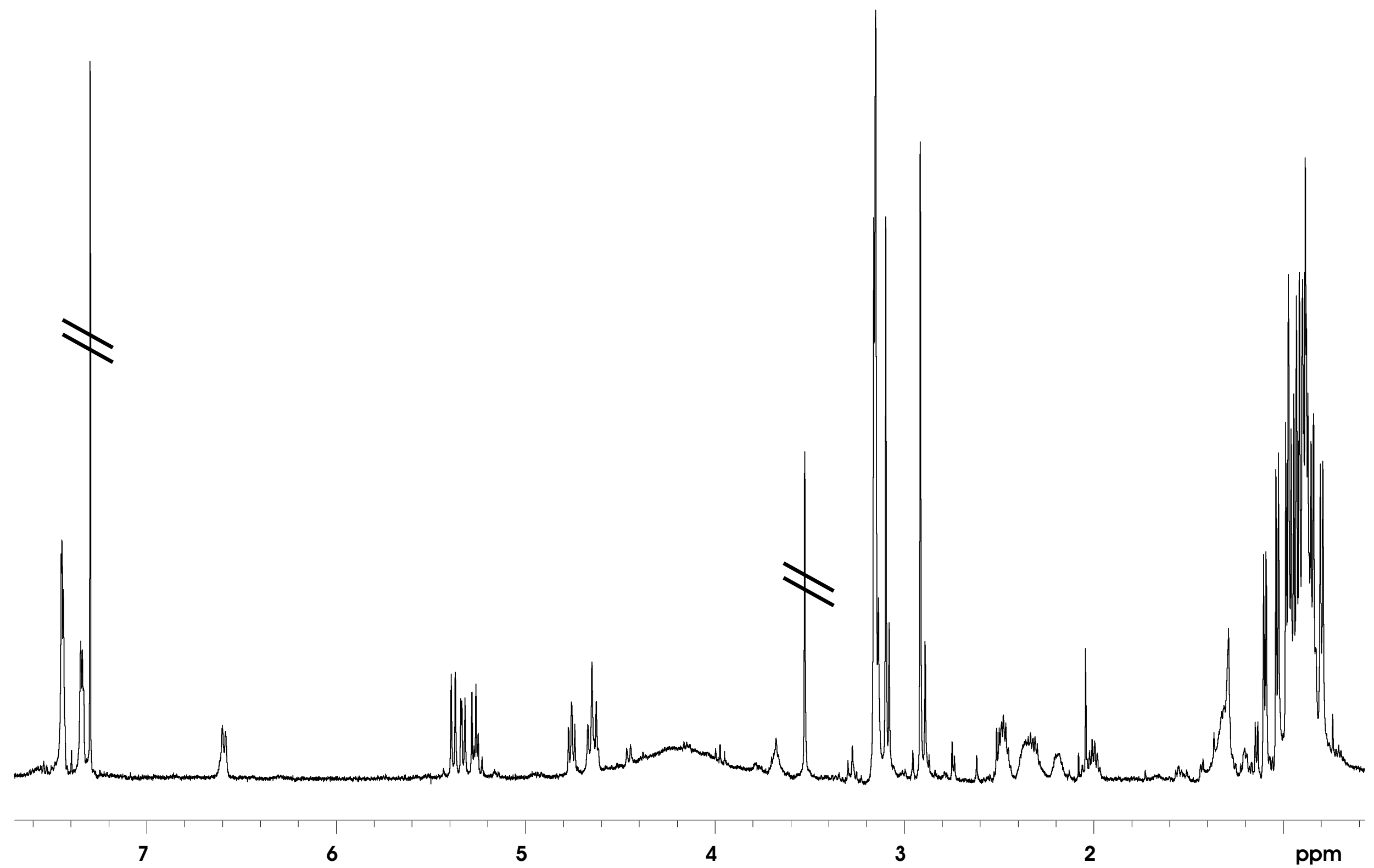

